\title{
Extreme Heat Fluxes in Gyrokinetic Simulations: a New Critical Beta
}

\author{
M.J. Pueschel ${ }^{1}$, P.W. Terry ${ }^{1}$, F. Jenko ${ }^{2}$, D.R. Hatch ${ }^{2}$, W.M. Nevins ${ }^{3}$, T. Görler ${ }^{2}$, and D. Told ${ }^{2}$ \\ ${ }^{1}$ University of Wisconsin-Madison, Madison, Wisconsin 53706, USA \\ ${ }^{2}$ Max-Planck-Institut für Plasmaphysik, EURATOM Association, D-85748 Garching, Germany \\ ${ }^{3}$ Lawrence Livermore National Laboratory, Livermore, California 94551, USA
}

\begin{abstract}
A hitherto unexplained feature of electromagnetic simulations of ion temperature gradient turbulence is the apparent failure of the transport levels to saturate for certain parameters; this effect, termed here Non-Zonal Transition, has been referred to as the high- $\beta$ runaway. The resulting large heat fluxes are shown to be a consequence of reduced zonal flow activity, brought on by magnetic field perturbations shorting out flux surfaces.
\end{abstract}

While zonal flows in electrostatic plasma microturbulence have been studied in detail [1-3], questions remain regarding the influence of magnetic fluctuations. As will be shown, under certain circumstances, such fluctuations can have a severe impact on turbulent saturation. Multiple gyrokinetic studies [4-7] demonstrate good agreement between codes for Cyclone Base Case (CBC) [8] parameters (which constitute a standard benchmark scenario) at finite normalized electron pressure $\beta$. Once $\beta$ exceeds a value of $0.8 \%$, however, heat fluxes do not appear to saturate anymore and instead grow to very large values. This has been termed runaway, but until now no encompassing explanation existed, and many approaches were discussed only informally. While the effect was mostly conjectured to stem from numerical shortcomings, Ref. [9] first suggested that it might be physical. Various codes - for standard initial conditions; see a comment in Ref. [10] - agree on the threshold [11] which shall be referred to as $\beta_{\text {crit }}^{\mathrm{NZT}}$, as opposed to the Kinetic Ballooning Mode (KBM) threshold $\beta_{\text {crit }}^{\mathrm{KBM}}$ (the point where KBMs become linearly unstable, close to where they become the dominant instability [7]); NZT stands for Non-Zonal Transition, a process which will be explored in some detail here. The NZT (KBM) threshold is extracted from simulations through a nonlinear (linear) $\beta$ scan. An NZT has also been observed in pure Ion Temperature Gradient (ITG) turbulence [10], as opposed to the CBC where Trapped Electron Modes (TEMs) play a role. It is to be stressed that if $\beta_{\text {crit }}^{\mathrm{NZT}}>\beta_{\text {crit }}^{\mathrm{KBM}}$, no effect is observed, as simulations of KBM turbulence tend not to remain stably saturated for long times.

A better understanding of this process is desirable since $\beta$ thresholds may limit the efficiency of fusion devices, but also because an unexplained effect of this severity may call into question the fundamentals of gyrokinetics. After a few words on the computational setup, the runaway phase is described, and the threshold is studied in more detail. It is shown that the NZT is caused by field line decorrelation which significantly enhances the level of flux-surface-breaking magnetic fluctuations. The impact of perturbed magnetic fields on zonal flows is studied through simulations, completed by a physical explanation which involves the impact of resonant mag-

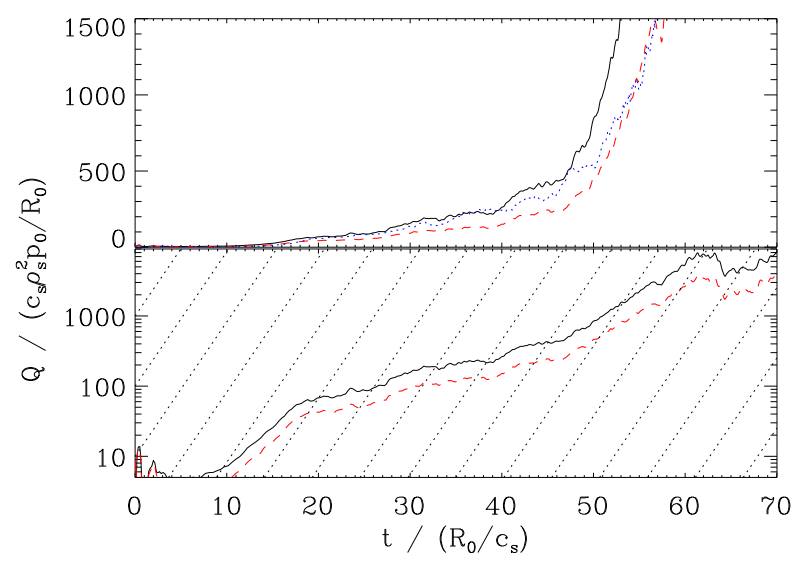

FIG. 1. (Color online) Transport levels $Q$ for $\beta=0.9 \%$ : the black solid, red dashed, and blue dotted lines correspond to the ion and electron electrostatic fluxes, and the electron electromagnetic flux, respectively. Both plots show the same data, the lower on a logarithmic axis, with black dotted lines of a slope twice the maximum linear growth rate, which is not exceeded by the $Q$ s.

netic fluctuations on zonal flows, based on an analytical model.

All simulations were performed with the GENE code $[12,13]$ in its radially local version. Details on the CBC-relevant (physical and numerical) parameters can be found in Ref. [7]. The NZT proves to be very robust with respect to numerical parameters and resolutions, which is why for some simulations, a slightly reduced grid was used, with $(128,16,16,32,8)$ modes/points in the radial, binormal, parallel, parallel velocity, and magnetic moment directions, respectively. These values have only a very moderate impact on the transport levels, do not affect the physics under investigation, and are very similar to the resolutions used in Ref. [6].

Fig. 1 shows fluxes during and after the runaway phase at $\beta=0.9 \%$. After initial saturation at $t \sim$ $(20-40) R_{0} / c_{\mathrm{s}}$ (the length of which is determined by the initial condition), growth quickly sets in again, and fluxes rise in a vaguely exponential manner (with growth not exceeding the maximum linear rate $\gamma_{\mathrm{ITG}}$ ), causing the time step to fall and making continuation of the sim- 


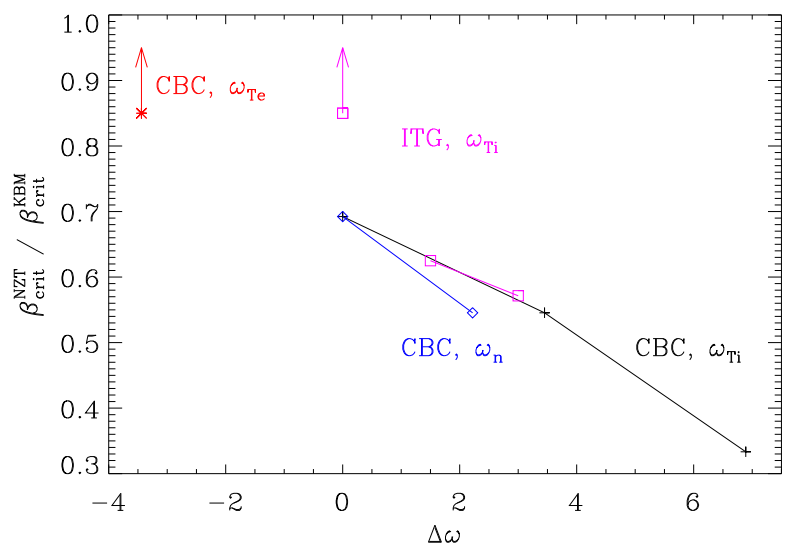

FIG. 2. (Color online) Impact of gradient variations $\Delta \omega$ on $\beta_{\text {crit }}^{\mathrm{NZT}}$ (normalized to $\beta_{\mathrm{crit}}^{\mathrm{KBM}}$ ) for CBC and ITG case parameters. Different symbols/colors indicate different gradients. Vertical arrows signify that for a particular $\Delta \omega$, the NZT threshold lies above the ballooning threshold.

ulation rather expensive.

At some point, however, simulations will saturate again at very large transport - this second saturation regime is of no consequence to experimental devices but makes numerical analyses like determining nonlinear frequencies or quasilinear ratios more convenient. Such analyses, both during the growth period and the second saturated phase, confirm that the runaway phenomenon is simply an ITG mode growing without the zonal flow being able to bring about its saturation: the nonlinear frequencies match the linear ITG, the transport and amplitude spectra hardly change qualitatively from those at lower $\beta$, but the zonal flow now has very little impact, and streamer-like structures dominate the perpendicular plane once the simulation has saturated again, strongly resembling the picture found for Electron Temperature Gradient driven turbulence $[12,14]$. When using a larger perpendicular box up to four times as big on each side as the default choice, the flux levels during the second saturation phase are lowered somewhat, but still orders of magnitude higher than those at lower $\beta$.

For a variety of reasons, it is preferable to replace the term runaway with non-zonal transition, and to refer to $\beta_{\text {crit }}^{\mathrm{NZT}}$ as the NZT threshold - this nomenclature will be used throughout this paper.

Known cases showing an NZT all exhibit background gradients larger than typically observed in experiments. The dependence of $\beta_{\text {crit }}^{\mathrm{NZT}}$ on the background gradients $\omega_{n, T}=R_{0} / L_{n, T}$ was thus assessed, where $R_{0}$ is the major radius and $L_{n, T}$ denotes the gradient scale length of the background temperature or density. Fig. 2 shows that $\beta_{\text {crit }}^{\mathrm{NZT}}$ depends more strongly on those gradients than does $\beta_{\text {crit }}^{\mathrm{KBM}}$, and that increasing gradients will lower the NZT relative to the KBM threshold. Combined, these dependencies resemble some features of the ITG (stronger drive

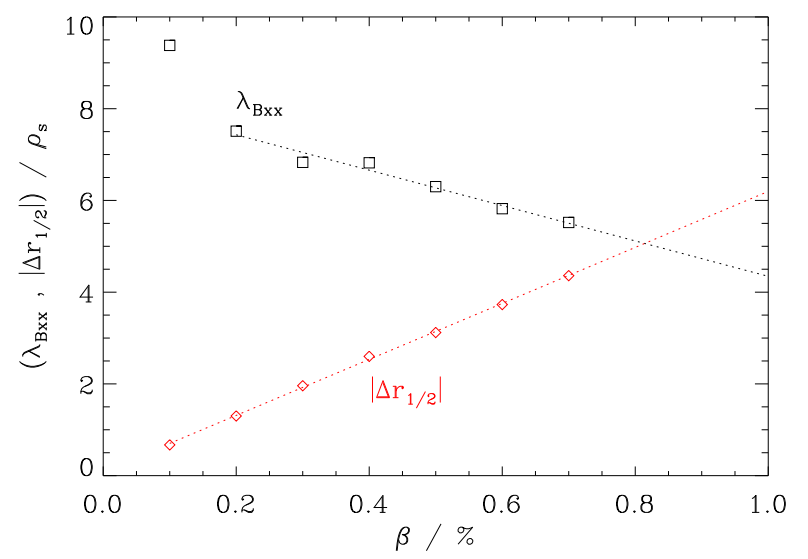

FIG. 3. (Color online) Half-turn radial field line displacement $\Delta r_{1 / 2}$ and $B_{x}$ correlation length $\lambda_{B x x}$ as a function of $\beta$; linear fits indicate an intersection just above $\beta=0.8 \%$.

for higher $\omega_{T \mathrm{i}}$ and $\omega_{n}$ ) as well as some of microtearing (stronger drive for higher $\omega_{T \mathrm{e}}$ and $\omega_{n}$ ) [15].

The NZT appears in some sense to be nearly marginal at standard CBC parameters (i.e., the NZT and the KBM threshold are of a similar magnitude), showing sometimes significant stretches of low-flux saturation before transitioning to high fluxes.

This leads to the central question of what brings about this change in the ITG-zonal-flow balance. No changes in the linear physics are observed near $\beta_{\mathrm{crit}}^{\mathrm{NZT}}$, such as the occurrence of new (subdominant but unstable) modes, phase or frequency shifts, or quasilinear flux ratios. A secondary instability analysis - measuring the growth of secondary modes destabilized through the nonlinear action of the linear mode - was performed, again revealing no significant changes of the nonlinear zonal flow drive between $\beta=0.7 \%$ and $0.9 \%$. In Ref. [9], subcritical excitation of KBMs as a tertiary instability (which in turn is excited nonlinearly by the secondary mode) due to nonlinear profile corrugations of temperature and density fluctuations was put forward as a possible cause. While the analyses in that paper are interesting and important in their own right they do not apply here: the corrugation amplitudes near $\beta_{\text {crit }}^{\mathrm{NZT}}$ are far too small to destabilize KBMs subcritically at this $\beta$; moreover, Ref. [9] ignores corrugations of the electrostatic potential-GENE simulations show that they negate much of the effect of the pressure corrugations via shear flow; suggesting that no subcritical KBM destabilization is possible for CBC parameters. In addition to this tertiary instability analysis, the nonlinear excitation of stable linear eigenmodes was studied for the present work. An investigation of the $\sim 10^{4}$ modes with the least negative growth rate revealed practically no changes at $\beta_{\text {crit }}^{\mathrm{NZT}}$ with respect to the relative distribution of nonlinear amplitudes, even taking into account the vastly different fluxes during the second saturation regime. More details on the analyses men- 
tioned here, as well as on nonlinear energy transfer, will be published in a separate paper. Regarding the latter, one can compute the $\left(k_{x}^{\prime}\right.$-averaged) nonlinear transfer $\mathcal{N}$ from $k_{y}$ to $k_{y}^{\prime}[16]$ : The result for $k_{y}^{\prime \prime}=k_{y}-k_{y}^{\prime}=0$, normalized to the sum over all interactions with $k_{y}^{\prime \prime} \neq 0$, constitutes a good measure for zonal flow coupling. Looking at this quantity in the range of $0<k_{y} \leq 0.35$ (and $k_{x}=0$ ), one finds that $\mathcal{N}\left(k_{y}, \beta=0.7 \%\right)<0$ (indicating that the zonal flow facilitates transfer to higher $k_{x}$, consistent with the conventional zonal flow shearing paradigm); whereas $\mathcal{N}\left(k_{y}, \beta=0.9 \%\right)>0$ (indicating that the zonal flow does not contribute to saturation), with $\langle\mathcal{N}(\beta=0.9 \%)\rangle_{k y} \approx 0.3\left|\langle\mathcal{N}(\beta=0.7 \%)\rangle_{k y}\right|$. In other words, zonal flow coupling is fundamentally changed once $\beta_{\text {crit }}^{\mathrm{NZT}}$ is exceeded.

This picture is confirmed by a closer look at the shearing rate $\omega_{\mathrm{s}}=\left\langle k_{x}^{2} \Phi_{\mathrm{fs}}\right\rangle$, where $\Phi_{\mathrm{fs}}$ is the flux-surface average of $\Phi$. This quantity parameterizes the effect of zonal flows, regardless of whether they regulate turbulence by energy transfer to stable modes or by shearing. It increases from $\sim 2$ during transient saturation to around $3-4$ afterwards; however, the root-mean-square $\Phi_{\text {rms }}$ increases by many orders of magnitude during the same time span, meaning the shearing rate - and thus the zonal flow - becomes less and less important.

Recently, it has been shown that (radial) magnetic fluctuations $B_{x}$ are composed of a quasilinear odd-parity component and an even-parity component, the latter of which is brought about by a subdominant microtearing mode $[16,17]$. Therefore, turbulent magnetic fields tend to contain some resonant (even parity) $B_{x}$ components which are able to break flux surfaces. As will be demonstrated, the non-resonant (odd-parity) $B_{x}$ fluctuations which are excited as part of the linear ITG mode can also become flux-surface-breaking under certain conditions - while usually not considered as a cause for magnetic stochasticity, this effect can play a crucial role when $B_{x}$ is spatially incoherent: Consider a field line subjected to an odd-parity $B_{x}$. Following this field line poloidally from $-\pi$ around the torus, one finds it to be radially displaced by a distance $\Delta r_{1 / 2}=r(0)-r(-\pi)$ from the unperturbed flux surface after half a poloidal turn. The mean displacement over the second half turn is then $\Delta r_{2 / 2} \approx-\Delta r_{1 / 2} \mathcal{C}_{B x x}\left(\Delta r_{1 / 2}\right)$, where it is sufficient here to approximate the radial correlation function of $B_{x}$ as being parabolic in $\Delta r / \lambda_{B x x}$ at small and vanishing at large separation, $\mathcal{C}_{B x x}(\Delta r) \approx \max \left[1-\left(\Delta r / \lambda_{B x x}\right)^{2}, 0\right]$; with the radial correlation length of $B_{x}$ denoted by $\lambda_{B x x}$, which describes the radial displacement where the autocorrelation function of $B_{x}$ (taken at the outboard midplane) has decreased to 0.368 . Consequently, the squared radial displacement after a full turn becomes

$$
\begin{aligned}
\left(\Delta r_{1 / 2}+\Delta r_{2 / 2}\right)^{2} & \approx 2 \Delta r_{1 / 2}^{2}\left(1-\mathcal{C}_{B x x}(\Delta r)\right) \\
& \approx 2 \Delta r_{1 / 2}^{2} \min \left[\left(\Delta r_{1 / 2} / \lambda_{B x x}\right)^{2}, 1\right] .
\end{aligned}
$$

When $\Delta r_{1 / 2}<\lambda_{B x x}$, the field line returns to its original position, thereby preserving flux surfaces. As $\Delta r_{1 / 2} \sim$ $\lambda_{B x x}$, however, the field line decorrelates from $B_{x}$ after its first half turn, and $\Delta r_{2 / 2}$ will no longer cancel $\Delta r_{1 / 2}$. Note that, in general, $\Delta r_{1 / 2}$ is determined by both the resonant and the non-resonant components of $B_{x}$. Fig. 3 shows that for CBC parameters, the first (i.e., most displaced) field lines reach the decorrelation condition $\Delta r_{1 / 2} \sim \lambda_{B x x}$ very near $\beta_{\text {crit }}^{\mathrm{NZT}}$. The sharp threshold for the NZT can be understood by considering the behavior of the stochastic radial conductivity $\sigma_{x} \propto D_{\mathrm{m}}$, where $D_{\mathrm{m}} \approx\left\langle\Delta r_{1 / 2}+\Delta r_{2 / 2}\right\rangle^{2} /\left(2 \pi q_{0} R_{0}\right)$ is the magnetic diffusivity [18]. With $\Delta r_{1 / 2} \propto \beta$ and using Eq. (1), it can be seen that $\sigma_{x} \propto \beta^{4}$ for $\beta<\beta_{\text {crit }}^{\mathrm{NZT}}$ in the case of purely non-resonant $B_{x}$. Once the threshold is crossed, the increase becomes less pronounced with a scaling of $\sigma_{x} \propto \beta^{2}$.

The occurrence of an NZT in the ITG case [10] (where $\omega_{T \mathrm{e}}=0$ and $B_{x}$ is almost entirely non-resonant) demonstrates that the threshold can exist without help from resonant $B_{x}$. The sensitivity of the NZT threshold to $\omega_{T \mathrm{i}}$ can be explained by the dependence of $B_{x}$ on the total heat diffusivity [19], which for ITG turbulence depends strongly on $\omega_{T i}$. To illustrate the consequences of zonal flows being subjected to magnetic fluctuations, a study of their corresponding impact is presented below.

Zonal flows are unstable to Geodesic Acoustic Modes (GAMs). After these have decayed away, the resulting residual state is stable in the absence of collisions $[1,2]$. For such simulations, background gradients and magnetic shear are turned off, and only one finite $\left|k_{x}\right|$ is included. When adding a binormal $B_{y}$ (self-consistently or as a constant), the residual level remains unchanged; the same goes for a non-resonant radial $B_{x}\left(k_{x}=0\right)$, e.g.: $B_{x} \propto \sin z$, where $z$ is the parallel coordinate. Only when adding a resonant $B_{x}-$ e.g.: $B_{x} \propto z^{0}$ - does the picture change, see Fig. 4 (here, $B_{x}$ is time-independent): while the GAM oscillations (not shown) do not feel its impact, the residual level sees a quadratic decrease with time. Physically, the electrons peel off their flux surface radially through parallel motion along perturbed field lines, creating radial currents which weaken the zonal flow. Practically, collisions or hyperdiffusion can be used to dampen the GAMs more quickly and obtain a cleaner residual; alternatively, the initial condition can be modified to result in a residual with near-Maxwellian ions, yielding the same effect at lower simulation cost.

The point $t_{\Phi=0}$ depends only on the resonant component of $B_{x}$, and can be parameterized (with $q_{0}$ denoting the safety factor and $\epsilon_{\mathrm{t}}$ the inverse aspect ratio) via $t_{\Phi=0} \propto q_{0}\left(1-\epsilon_{\mathrm{t}}\right) B_{x}^{-1}$, independently of the zonal flow $k_{x}$-thorough tests were performed to ensure numerical convergence. Note that while this study focuses on resonant $B_{x}$, it is equally applicable to non-resonant but flux-surface-breaking fluctuations in the context of the NZT.

Instead of a constant amplitude, $B_{x}$ can be chosen to 
scale with the spatially averaged $|\Phi|$, thus creating a decay resembling an exponential curve which depends on the various physical parameters in a similar way as the above study. This imitates the turbulent scenario more closely, but does not include the destabilizing reaction of the ITG turbulence to the diminished zonal flow. In summary, these zonal flow studies illustrate the destructive impact of a flux-surface-breaking $B_{x}$ on the zonal flows, corroborating the above description of the NZT. Next, these findings are compared to the results of an analytical model.

A constant-in-time, resonant $B_{x}\left(k_{x}=0\right)$ can be added to the calculations presented in Refs. [1, 2]. The full derivation has to be deferred to a separate publicationhere, only key assumptions and results will be shown. In such a magnetic field, electrons will leave a flux surface through parallel motion on a time scale depending on their individual $v_{\|}$. At short times, the ions do not (yet) respond to the electrons, and ambipolar effects are expected to be small. With $B_{x}$ entering solely through the source $S_{k}^{\Phi}$, a Laplace transform of $\Phi$ leads to

$$
\Phi(t)=\int_{0}^{t} \lambda\left(t^{\prime}\right) \kappa\left(t-t^{\prime}\right) \mathrm{d} t^{\prime}
$$

with

$$
\lambda(t)=\frac{\Phi(t=0)}{\mathcal{R}}-\frac{2 \int \mathrm{d} v_{\perp} F_{0}\left(v_{\perp}\right) S_{k}^{\Phi}\left(\mathrm{e}^{\alpha^{2} t^{2}}-1\right)}{\left(n_{0} e / T_{\mathrm{i}}\right) k_{\perp}^{2} \rho_{s}^{2} \mathcal{R}},
$$

where the frequency-like parameter $\alpha=v_{\|} k_{x} B_{x} / B_{0}$ and the residual factor $\mathcal{R}=1+1.6 q_{0}^{2} / \epsilon_{\mathrm{t}}^{1 / 2}$ have been introduced, as well as the adiabatic response $\kappa(t)$ which involves poles for residue integration. The other quantities are the perpendicular velocity $v_{\perp}$ and the background distribution $F_{0}$. For short times $t$, the form of $\lambda(t)$ will determine the behavior of $\Phi(t)$, as $\kappa(t) \rightarrow \delta(t)$. As expected, $\lambda(t)$ includes the usual residual, but the second term causes the potential to decrease quadratically in time, as $\exp \left(\alpha^{2} t^{2}\right)-1 \approx \alpha^{2} t^{2}$ for small $t$. In GENE normalization, $\alpha^{2} t^{2} \sim\left(m_{\mathrm{i}} / m_{\mathrm{e}}\right) k_{x}^{2} t^{2} B_{x}^{2} / B_{0}^{2}$, and thus the small-argument expansion holds over a large range of $t$ shown in Fig. 4 (here, $k_{y}=0.05$ ), consistent with the fact that the dotted curve provides an excellent fit for $t \lesssim 100 c_{s} / R_{0}$. The central features of the simulations have thus been recovered. The attention is now focused again on the overall explanation of the NZT.

The following consistent picture emerges: the ITG mode grows linearly and excites a zonal flow through which it saturates. If $\beta>\beta_{\text {crit }}^{\mathrm{NZT}}$, sufficiently strong, nonresonant $B_{x}$ fluctuations created by the linear mode then decorrelate field lines and cause the zonal flow to decay due to flux surfaces shorting out (with or without the help of resonant fluctuations), in turn allowing the ITG transport to increase. Therefore, the ITG mode will continue to grow, without the zonal flow being able to counteract

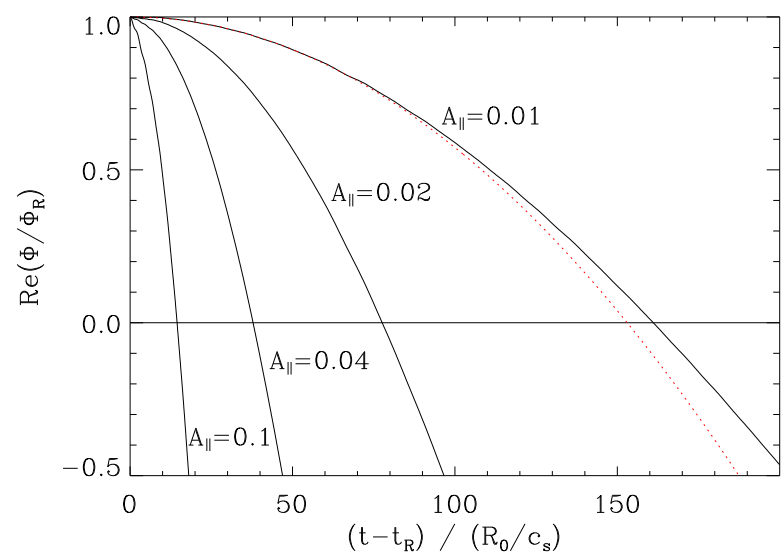

FIG. 4. (Color online) Zonal flow decay in the presence of various values of a resonant and time-independent $A_{\|} \propto B_{x}$. Shown is the real part of the electrostatic potential $\Phi$ relative to the residual $\Phi_{\mathrm{R}}$ (with $\operatorname{Im}(\Phi) \ll \operatorname{Re}(\Phi)$ ); time is measured relative to $t_{\mathrm{R}}$ where the residual had been reached $\left(B_{x}\right.$ is turned on $\left.t=t_{\mathrm{R}}\right)$. The dotted red line shows an exemplary quadratic fit to the data, consistent with analytical theory.

it sufficiently to saturate it, and the $B_{x}$ amplitude will grow in tandem. Once very large fluxes are reached, it can be conjectured that the turbulence saturates via a Kelvin-Helmholtz-type mechanism [12, 20]; little physical meaning can be attached to that phase, however. As a consequence of its zonal flow dynamics, TEM turbulence is likely not prone to an NZT. While for certain parameter regimes, zonal flows play some role in TEM saturation [21], the mode should be able to fall back on non-zonal saturation at similar or slightly larger transport values if the zonal flows are critically weakened.

In conclusion, a physical explanation for the phenomenon of the non-zonal transition has been provided, involving the reduction of zonal flow strength by radial magnetic fluctuations. Not only does this create a new $\beta$ limit which can be more restrictive than the KBM threshold for sufficiently large pressure gradients - larger, however, than those of typical present-day fusion experiments - but it may also open new approaches to plasma control: an external mechanism to suppress fluctuations would bolster the zonal flows and thus reduce transport.

The authors wish to acknowledge valuable discussions with C.C. Hegna, T. Dannert, D. Carmody, F. Hinton, R.E. Waltz, and J.-H. Kim.

[1] M.N. Rosenbluth and F.L. Hinton, Phys. Rev. Lett. 80, 724 (1998)

[2] F.L. Hinton and M.N. Rosenbluth, Plasma Phys. Control. Fusion 41, 653 (1999)

[3] P.H. Diamond et al., Plasma Phys. Control. Fusion 47, R35 (2005) 
[4] Y. Chen et al., Nucl. Fusion 43, 1121 (2003)

[5] S.E. Parker et al., Phys. Plasmas 11, 2594 (2004)

[6] J. Candy, Phys. Plasmas 12, 72307 (2005)

[7] M.J. Pueschel et al., Phys. Plasmas 15, 102310 (2008)

[8] A.M. Dimits et al., Phys. Plasmas 7, 969 (2000)

[9] R.E. Waltz, Phys. Plasmas 17, 072501 (2010)

[10] M.J. Pueschel and F. Jenko, Phys. Plasmas 17, 062307 (2010)

[11] W.M. Nevins et al., Turbulence-Driven Magnetic Reconnection, American Physical Society, 51st Annual Meeting of the APS Division of Plasma Physics (2009)

[12] F. Jenko et al., Phys. Plasmas 7, 1904 (2000)
[13] see http://gene.rzg.mpg.de for code details and access

[14] W.M. Nevins et al., Phys. Plasmas 13, 122306 (2006)

[15] H. Doerk et al., Phys. Rev. Lett. 106, 155003 (2011)

[16] D.R. Hatch et al., Phys. Plasmas 18, 055706 (2011)

[17] D.R. Hatch et al., Phys. Rev. Lett. 108, 235002 (2012)

[18] W.M. Nevins, E. Wang, and J. Candy, Phys. Rev. Lett. 106, 065003 (2011)

[19] M.J. Pueschel et al., Nucl. Fusion 52, 103018 (2012)

[20] P. Xanthopoulos et al., Phys. Rev. Lett. 99, 035002 (2007)

[21] D.R. Ernst et al., Phys. Plasmas 16, 055906 (2009) 